\title{
Spatial cuing does not affect the magnitude of the attentional blink
}

\author{
SHAHAB GHORASHI ANd JAMEs T. EnNS \\ University of British Columbia, Vancouver, British Columbia, Canada \\ AND \\ Thomas M. SpaleK and Vincent Di Lollo \\ Simon Fraser University, Burnaby, British Columbia, Canada
}

\begin{abstract}
Identification of the second of two targets is impaired when the second target is presented less than about $500 \mathrm{msec}$ after the first. Nieuwenstein, Chun, van der Lubbe, and Hooge (2005, Experiment 4) reported that the magnitude of this attentional blink (AB) is reduced when the location of the second target is precued. Here we show how that finding resulted from an artifact brought about by a ceiling imposed by data limitation. Instead of using an accuracy measure, the present work used a dynamic threshold-tracking procedure that was not constrained by a performance ceiling. The results show that, when the ceiling is removed, spatial cuing does not affect and is not affected by the $\mathrm{AB}$. These results are consistent with the hypothesis that cue localization and target identification may take place along separate (dorsal and ventral) visual pathways.
\end{abstract}

Attentional limitations in visual processing are revealed by a phenomenon known as the attentional blink (AB), in which the identification of the second of two targets (T2) is impaired when the second target is presented less than about 500 msec after the first target (T1; Raymond, Shapiro, \& Arnell, 1992). This deficit is commonly found to be most pronounced when the intertarget lag is short and to be diminished progressively as the lag is increased.

Several factors are known to influence the magnitude of the AB. For example, the deficit is much reduced when T2 is one's own name (Shapiro, Caldwell, \& Sorensen, 1997) or when the observer adopts a more relaxed approach to the task (Olivers \& Nieuwenhuis, 2005). Whether cuing also affects the magnitude of the $\mathrm{AB}$ has been investigated by Nieuwenstein, Chun, van der Lubbe, and Hooge (2005). They set out to find out whether the magnitude of the AB is reduced when $\mathrm{T} 2$ is preceded by a cue that shares the target's defining characteristic or, equivalently, by a cue that indicates the location of an upcoming target.

On the basis of the results of four experiments, Nieuwenstein et al. (2005) concluded that the cuing of 22 reduces the magnitude of the $\mathrm{AB}$. That conclusion is questioned in the present work. We argue that Nieuwenstein et al.'s results were vitiated by a performance ceiling imposed by data limitation. The problem is perhaps best exemplified in Nieuwenstein et al.'s Experiment 4. In that experiment, the basic display consisted of four square outlines that acted as place holders for the stimuli above, below, left, or right of a central fixation cross. The display sequence began with
T1 (a letter), which was displayed briefly inside a randomly chosen placeholder and followed immediately by a mask. T2, which was also followed by a mask, was presented inside one of the three remaining placeholders at random after an intertarget lag that was either short $(306 \mathrm{msec})$ or long (706 msec). It is important to note that a spatial cue ("+") was presented in the T2 placeholder $94 \mathrm{msec}$ before the onset of T2. Each observer served in a preliminary experiment aimed at keeping performance well below the 100\% ceiling imposed by the response scale. In that experiment, a single target was followed by a mask. The relative exposure durations of the target and the mask were varied systematically within a total duration of $106 \mathrm{msec}$; as the duration of the target was increased, the duration of the mask was decreased correspondingly. The specific combination of target and mask durations that yielded $70 \%$ correct target identifications for each individual observer was used in the experiment proper as the combination of durations of T1 plus mask and T2 plus mask for that observer.

The results of Nieuwenstein et al.'s (2005) Experiment 4 are illustrated in Figure 1B. Cuing is seen to enhance accuracy of T2 identification at the short lag but not at the long lag. This result led to the conclusion that cuing reduces the magnitude of the $A B$.

On the face of it, the finding that cuing did not facilitate T2 identification at the longer lag (Figure 1B) seems contrary to the well-established finding that cuing the location at which a target is about to appear leads to substantial benefits (see, e.g., Colegate, Hoffman, \& Eriksen,

S. Ghorashi, ghorashi@psych.ubc.ca 
A

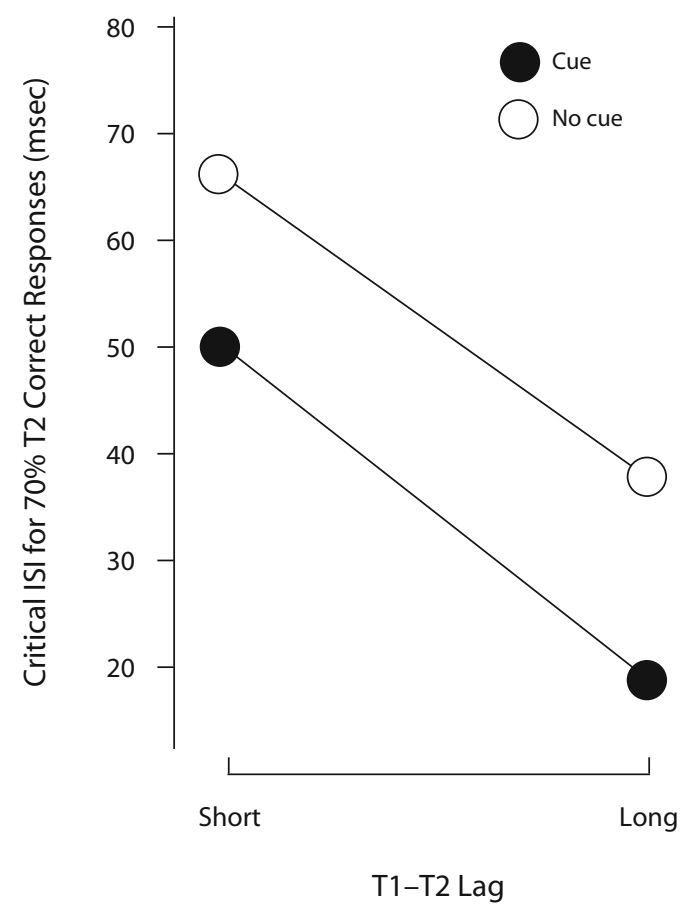

B

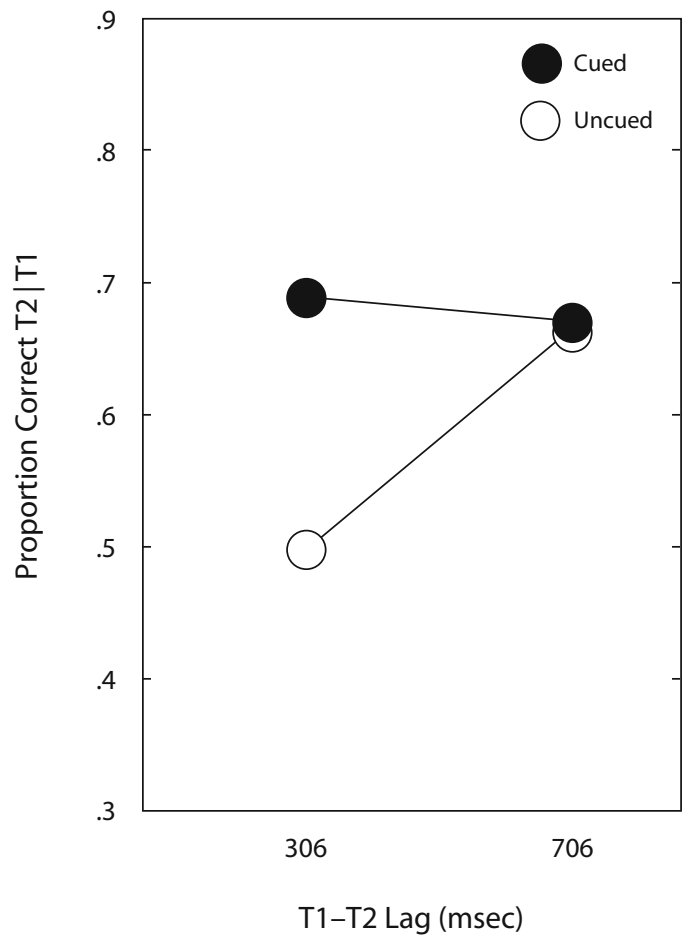

Figure 1. (A) Results of the present experiment. T1-T2 lag varied slightly across observers, depending on the duration of the ISI between T1 and the trailing mask, as determined in the preliminary procedure. Averaged across observers, the short lag was $318 \mathrm{msec}$, and the long lag was $717 \mathrm{msec}$. (B) Results of Experiment 4 of Nieuwenstein, Chun, van der Lubbe, and Hooge (2005). T1, first target; T2, second target; ISI, interstimulus interval.

1973). By the same token, it appears odd that a cue that is demonstrably ineffectual outside the period of the $\mathrm{AB}$ (lag $=706 \mathrm{msec})$ appears to become effectual within the period of the $\mathrm{AB}$ ( $\mathrm{lag}=306 \mathrm{msec}$ ), just when stimulus processing is supposed to be most impaired.

We claim that both of these problematic findings are likely to have arisen from a procedural artifact - namely, that by avoiding the $100 \%$ ceiling imposed by the response scale, the preliminary procedure implemented by Nieuwenstein et al. (2005) might have introduced a ceiling imposed by data limitation. The principal aim of the preliminary procedure was to impoverish a single target so that it could not be identified correctly more than $70 \%$ of the time. It is, therefore, not surprising that, in the experiment proper, T2 performance could not exceed that $70 \%$ level (Figure 1B). It is likely that the impoverishment was mediated by temporal-integration masking, in which the target and the mask were perceived as being a single compound stimulus. This degradation resulted in an effective data limitation that constrained performance to $70 \%$ not only for T2 (Figure 1B) but also for T1, which was identified correctly on $69 \%$ of the trials.

Like Nieuwenstein et al. (2005, Experiment 4), we investigated the effect of a spatial cue on the magnitude of the $\mathrm{AB}$. The present procedures, however, avoided a performance ceiling by employing a dynamic threshold-tracking procedure known as parameter estimation through sequen- tial testing (PEST; Taylor \& Creelman, 1967). The display sequence was the same as that in Nieuwenstein et al.'s study, except that T2 was displayed for only $13.3 \mathrm{msec}$ and was separated from the trailing mask by an interstimulus interval (ISI), during which the screen was blank. An ISI was inserted between the target and the mask in order to minimize the probability of temporal integration (Di Lollo, Hogben, \& Dixon, 1994). The duration of the ISI was varied dynamically by PEST separately for each observer in order to converge on a level of $70 \%$ correct $\mathrm{T} 2$ responses. The dependent measure was the critical ISI (ISI ${ }_{c}$ ), at which the observer obtained $70 \%$ correct responses. By its very nature, $\mathrm{ISI}_{\mathrm{c}}$ is free from ceiling constraints.

\section{Observers}

\section{METHOD}

Twenty-one undergraduate volunteers at Simon Fraser University participated for class credit or payment. All of the observers reported normal or corrected-to-normal vision and were naive about the purpose of the experiment.

\section{Apparatus and Stimuli}

The stimuli consisted of sequences of two uppercase letters chosen randomly without replacement on each trial from the English alphabet, but I, O, Q, and $\mathrm{Z}$ were not used. All of the stimuli were black, subtended approximately $1^{\circ}$ of visual angle, and were presented on a medium-gray background (approximately $42 \mathrm{~cd} / \mathrm{m}^{2}$ ) at a luminance of approximately $0.5 \mathrm{~cd} / \mathrm{m}^{2}$, as measured by a Minolta LS 100 luminance meter. The screen's refresh rate was $75 \mathrm{~Hz}$. The 
observers sat in a dimly lit room and viewed the displays from a distance of approximately $60 \mathrm{~cm}$.

\section{Procedure}

An overriding design consideration was to replicate, as closely as possible, the procedures used in Nieuwenstein et al.'s (2005) Experiment 4. The sequence of stimuli is illustrated in Figure 2. After the observer initiated a trial by pressing the space bar, the fixation cross disappeared from the center of the screen, and $\mathrm{T} 1$ was presented for $13.3 \mathrm{msec}$ in one of four screen locations, chosen randomly on each trial, at $2^{\circ}$ (center to center) above, below, to the left, or to the right of the fixation cross. T1 was followed by a blank ISI, which was followed by a mask consisting of two side-by-side \# signs displayed for $66.5 \mathrm{msec}$. The ISI between T1 and its mask was fixed throughout the experiment and was determined separately for each observer in a preliminary procedure, in which PEST was used to find the ISI at which the observer could identify a single target followed by the \#\# mask approximately $70 \%$ of the time. The mean ISI, averaged across observers, was $38.7 \mathrm{msec}(S D=16.3)$. Thus, on average, the exposure duration for T1, the ISI, and the mask was $118.5 \mathrm{msec}$.

The T1 mask was followed by a blank screen displayed for $106 \mathrm{msec}$ on half of the trials and for $505 \mathrm{msec}$ on the other half. On half of the trials in each of the two conditions, the blank screen was followed by a spatial cue ("+") for T2. The cue was displayed for $13.3 \mathrm{msec}$, followed by a blank screen for $80 \mathrm{msec}$. On trials in which the spatial cue was not displayed, the duration of the blank screen was increased to $93.3 \mathrm{msec}$. T2 was presented for $13.3 \mathrm{msec}$ in one of the remaining three screen locations (other than the T1 location) chosen randomly on each trial. T2 was followed by a blank ISI, which was followed by the \#\# mask for $66.5 \mathrm{msec}$. Thus, the two targets were separated either by a short stimulus onset asynchrony (SOA; lag $\sim 318 \mathrm{msec}$ ) or by a long SOA ( 717 msec).

The principal dependent variable in the present experiment was the duration of the ISI $I_{c}$ between T2 and its mask, at which any given observer could identify T2 approximately $70 \%$ of the time. The ISI between T2 and its mask was varied dynamically by PEST, which reduced the ISI when the observer's response accuracy exceeded the criterial level and increased it when accuracy was too low. A Wald (1947) sequential likelihood-ratio test determined whether the immediately preceding run of responses yielded an event proportion greater or less than $70 \%$. The Wald routine was called only on trials in which $\mathrm{T} 1$ had been identified correctly. The PEST end run consisted of 16 trials after three reversals in the direction of adjustment of the ISI had been recorded. The ISI $\mathrm{c}_{\mathrm{c}}$ was the mean ISI over those last 16 trials; thus, ISI $_{c}$ represents the duration of the mask-free interval after T2 offset that is necessary for achieving the criterial level of accuracy, separately for each observer. The observers were required to report both targets in any order-guessing, if unsure. ${ }^{1}$ Thus, the design was a 2 (T1-T2 lag: short or long) $\times 2$ (cue: present or absent) factorial.

\section{RESULTS AND DISCUSSION}

The mean percentages of correct responses for T1, averaged over lags, were $59.7 \%$ and $57.9 \%$ for the cue and no-cue conditions, respectively. A 2 (lag: short or long) $\times$ 2 (cuing: cue or no cue) repeated measures ANOVA performed on the T1 scores revealed no significant effects of lag or cuing (both $F_{\mathrm{S}}<1$ ) and no significant interaction $[F(1,20)=1.41, p=.25]$. The ISI $\mathrm{C}_{\mathrm{c}} \mathrm{s}$ were averaged across observers, separately for each lag and cuing condition, and are illustrated in Figure 1A. The scores were analyzed in a 2 (lag: short or long) $\times 2$ (cuing: cue or no cue) repeated measures ANOVA, which revealed significant effects of $\operatorname{lag}[F(1,20)=19.22, p<.001]$ and cuing $[F(1,20)=6.19$, $p=.02]$. The interaction was not significant $(F<1)$.

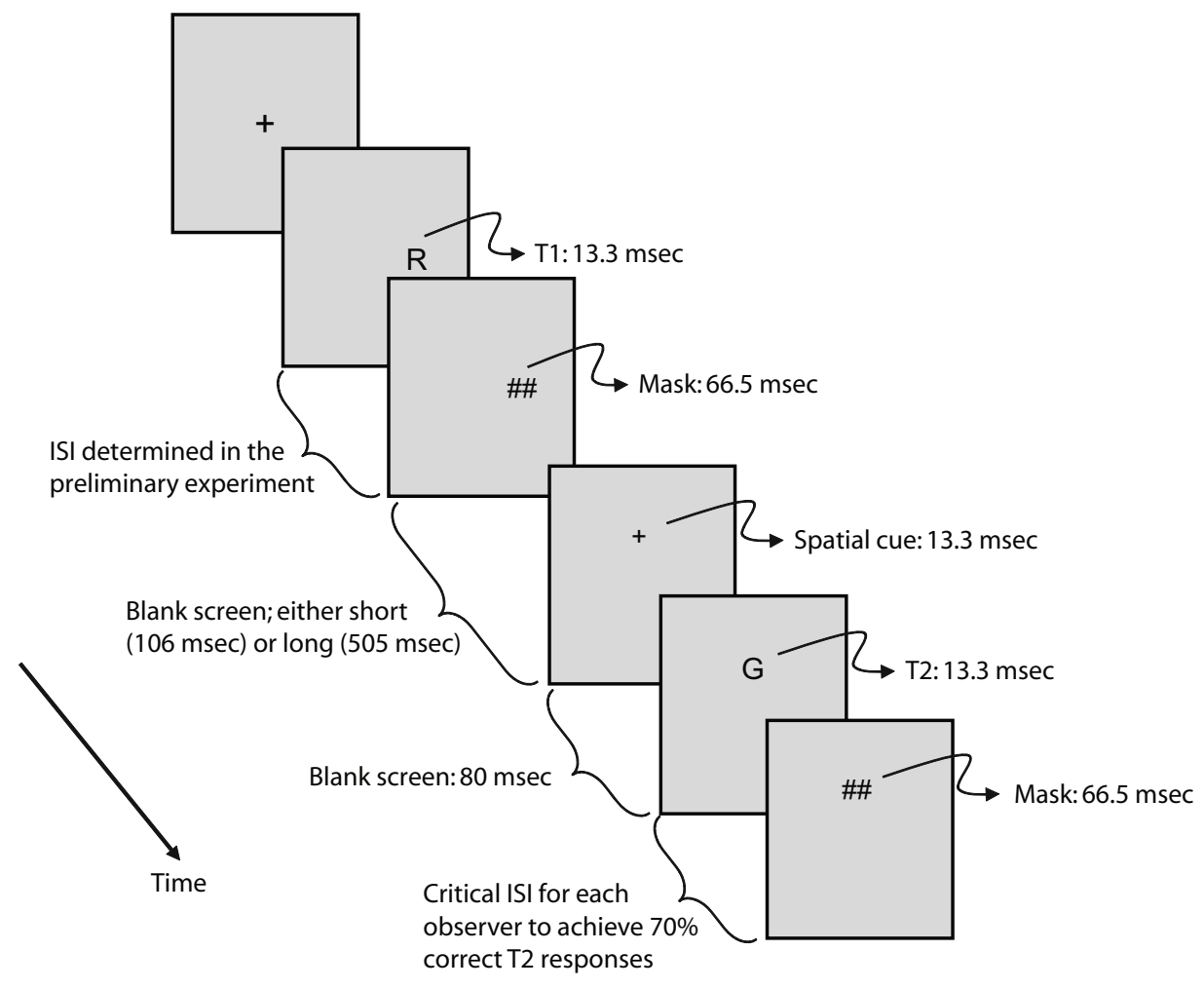

Figure 2. Sequence of events on each trial. On this trial, T2 (second target) was cued. T1, first target; ISI, interstimulus interval. 
The PEST procedure avoids ceiling effects, but it is open to possible floor effects that might prevent an interaction from being evident. A floor effect could occur if the ISI between T2 and its mask is decreased to the irreducible minimum of zero refresh frames (i.e., $\mathrm{ISI}_{\mathrm{c}}=0$ ). An $\mathrm{ISI}_{\mathrm{c}}$ equal to zero was obtained by 2 observers at the long intertarget lag in the cue condition and by 2 observers in the long intertarget lag in the no-cue condition. The results of an ANOVA performed on the data that did not include those observers were virtually identical to those found in the earlier analysis. The analysis revealed significant effects of lag $[F(1,16)=10.81, p<.005]$ and cuing $[F(1,16)=13.67, p=.002]$. The interaction was again not significant $(F<1)$. Thus, possible floor effects were not a consideration in the present experiment.

Spatial cuing enhanced T2 performance, but it did so equally across lags (Figure 1A); that is, the cue enhanced T2 performance to the same extent whether T2 was presented during the period of the $\mathrm{AB}$ or beyond it. This means that spatial cuing and the $\mathrm{AB}$ are independent effects. The present findings strongly suggest that Nieuwenstein et al.'s (2005, Experiment 4) conclusion-that cuing reduces the magnitude of the $\mathrm{AB}$ - was based on an artifact brought about by a ceiling imposed by data limitation. When the ceiling is removed, as was done in the present work, the results show that spatial cuing does not affect and is not affected by the AB.

It must be noted that Nieuwenstein et al. (2005) were not unaware of a data limitation in their experiments: "Cuing did not enhance report ... when performance was data limited in a dual-target condition" (p. 1470). What they did not consider was that it might have been impossible for T2 performance to exceed the ceiling imposed by the data limitation whether or not T2 was precued or whether it was presented during the period of the $\mathrm{AB}$ or beyond it. The finding that a leading cue did not facilitate the report of a trailing target outside the period of the $\mathrm{AB}$ is itself remarkable because it is at odds with the established finding in the spatial cuing literature. That finding ceases to be anomalous, however, when it is seen as being the result of a ceiling imposed by data limitation.

Nieuwenstein et al.'s (2005) results led to two major conclusions. First, "The AB can be markedly reduced or even fully prevented when T2 is precued" (p. 1473). This conclusion is disconfirmed by the present results (Figure 1A), which point instead to a performance ceiling as the critical factor. And second, in agreement with predictions from the delayed attentional engagement (DAE) hypothesis (Nieuwenstein et al., 2005), "Cuing appears to counteract a delay in the selection of potential targets for consolidation" (p. 1473). In a restricted sense, this conclusion is valid and consistent with the outcomes of the present work and with the conventional finding that cuing facilitates target processing. However, the present finding - that the extent of cuing-linked facilitation is invariant with intertarget lag — cannot be explained by the DAE hypothesis, in which cuing is said to be effective only during the period of the $\mathrm{AB}$.

There are several potential accounts for the independence of cuing and the $\mathrm{AB}$ seen in the present work. One plausible option is that cue localization and target identification may take place along distinct processing pathways. Spalek, Falcon, and Di Lollo (2006) have proposed a hybrid input-filtering model in which different classes of stimuli are processed along separate pathways. Salient stimuli, such as the spatial cue employed in the present study, are said to gain direct access to high-level processing along a dedicated pathway that is free from the $\mathrm{AB}$ bottleneck.

A dual-pathway account has also been proposed by Ghorashi, Enns, and Di Lollo (2008), in terms of the distinction between dorsal and ventral visual pathways (Ungerleider \& Mishkin, 1982). Ghorashi et al. have proposed that the independence of spatial-selection and identity-extraction processes can be mapped directly on the functional distinction between dorsal and ventral streams. Specifically, they have suggested that spatial selection is carried out mainly along the dorsal pathway and that target identification is carried out principally along the ventral pathway. Within this conceptual framework, cuing was not affected by the $\mathrm{AB}$ in the present work because spatial selection and identity extraction are mediated by processing mechanisms that are anatomically and functionally distinct.

Whether nonspatial cuing is also independent of the $A B$ remains to be determined. A suggestion of independence is provided by the outcome of Nieuwenstein et al.'s (2005) Experiment 2. In that experiment, all stimuli were presented in rapid serial visual presentation in the center of the screen. The distractors were black letters and the targets were colored digits. In the cuing conditions, T2 was reliably preceded by a cue in the form of two distractors that were colored either the same as or different from T2. In a no-cue condition, $\mathrm{T} 2$ was preceded by black distractors. The finding of principal interest to us was that the functions for the different-color cue and no-cue conditions, while differing from one another in level, were parallel, like the functions seen in Figure 1A. This suggests that cuing may not affect the magnitude of the $A B$, even when the cuing is nonspatial. Further experimentation is clearly required, however, in order to reach an unambiguous conclusion.

One last issue needs to be raised. In the present work, the abrupt onset of the spatial cue caused attention to be driven exogenously. Under these conditions, cuing was not impaired during the $\mathrm{AB}$. Whether or not cuing would remain unimpaired under conditions in which attention is driven endogenously is uncertain. On one hand, Dell'Acqua, Sessa, Jolicœur, and Robitaille (2006) have reported that the endogenous control of attention is impaired during the AB. On the other hand, Zhang, Shao, Nieuwenstein, and Zhou (2008) have found that endogenous control is not impaired during the $\mathrm{AB}$. A resolution of this issue, however, is beyond the scope of the present study.

\section{AUTHOR NOTE}

The present study was supported by a PGS-D grant from the Natural Sciences and Engineering Research Council of Canada to S.G. and by Discovery grants from the Natural Sciences and Engineering Research Council of Canada to J.T.E., T.M.S., and V.D.L. Correspondence concerning this article should be addressed to S. Ghorashi, Department of 
Psychology, University of British Columbia, 2136 West Mall, Vancouver, BC, V6T 1 Z4 Canada (e-mail: ghorashi@psych.ubc.ca).

\section{REFERENCES}

Colegate, R. L., Hoffman, J. E., \& Eriksen, C. W. (1973). Selective encoding from multielement visual displays. Perception \& Psychophysics, 14, 217-224.

Dell'Acqua, R., Sessa, P., Joliceur, P., \& Robitaille, N. (2006). Spatial attention freezes during the attention blink. Psychophysiology, 43, 394-400.

Di Lollo, V., Hogben, J. H., \& Dixon, P. (1994). Temporal integration and segregation of brief visual stimuli: Patterns of correlation in time. Perception \& Psychophysics, 55, 373-386.

Ghorashi, S., EnNS, J. T., \& Di Lollo, V. (2008, November). Spatial orienting is not impaired during the attentional blink. Abstracts of the Psychonomic Society, 49, 55.

Nieuwenstein, M. R., Chun, M. M., van der Lubbe, R. H. J., \& Hooge, I. T. C. (2005). Delayed attentional engagement in the attentional blink. Journal of Experimental Psychology: Human Perception \& Performance, 31, 1463-1475.

Olivers, C. N. L., \& Nieuwenhuis, S. (2005). The beneficial effect of concurrent task-irrelevant mental activity on temporal attention. Psychological Science, 16, 265-269.

Raymond, J. E., Shapiro, K. L., \& Arnell, K. M. (1992). Temporary suppression of visual processing in an RSVP task: An attentional blink? Journal of Experimental Psychology: Human Perception \& Performance, 18, 849-860.

Shapiro, K. L., Caldwell, J., \& Sorensen, R. E. (1997). Personal names and the attentional blink: A visual "cocktail party" effect. Journal of Experimental Psychology: Human Perception \& Performance, 23, 504-514.

SpaleK, T. M., Falcon, L. J., \& Di Lollo, V. (2006). Attentional blink and attentional capture: Endogenous versus exogenous control over paying attention to two important events in close succession. Perception \& Psychophysics, 68, 674-684.
Taylor, M. M., \& Creelman, C. D. (1967). PEST: Efficient estimates on probability functions. Journal of the Acoustical Society of America, 41, 782-787.

Ungerleider, L. G., \& Mishrin, M. (1982). Two cortical visual systems. In D. J. Ingle, M. A. Goodale, \& R. J. W. Mansfield (Eds.), Analysis of visual behavior (pp. 549-586). Cambridge, MA: MIT Press.

WALD, A. (1947). Sequential analysis. New York: Wiley.

Zhang, D., Shao, L., Nieuwenstein, M. [R.], \& Zhou, X. (2008). Topdown control is not lost in the attentional blink: Evidence from intact endogenous cuing. Experimental Brain Research, 185, 287-295.

\section{NOTE}

1. This scoring procedure matched that used in the study of Nieuwenstein et al. (2005, Experiment 4). We thank M. R. Nieuwenstein for supplying this information (personal communication, January 2009). An alternative procedure is to score the responses as correct only when the two targets are reported in the correct order. Direct comparison shows that accuracy of T2 identification is lower in the ordered than in the not-ordered procedure, with the difference being largest when T2 is presented directly after T1 (i.e., at lag 1; Spalek, Falcon, \& Di Lollo, 2006). In the study by Spalek et al., T2 accuracy at lag 1 was $83.0 \%$ and $55.8 \%$ for the not-ordered and the ordered procedures, respectively. This difference of $27.2 \%$ occurred because, in the not-ordered procedure, there is only one relevant source of impairment: whatever causes the $\mathrm{AB}$ deficit itself. In contrast, in the ordered procedure, there is an additional source of impairment, which is confounded with the first: loss of temporalorder information when the two targets are presented in rapid succession. The two sources seem to combine additively, as revealed by the finding that the difference in accuracy between the ordered and not-ordered procedures is virtually the same for T1 $(29.1 \%)$ as for T2. The not-ordered procedure is, therefore, the procedure of choice if the $\mathrm{AB}$ deficit is to be estimated separately from the loss of temporal-order information.

(Manuscript received January 5, 2009; accepted for publication January 12, 2009.) 